\title{
Integrity of the pheochromocytoma susceptibility TMEM127 gene in patients with pediatric malignancies
}

\section{Dear Editor,}

Germline mutations of the tumor suppressor gene TMEM127 occur in the neural-crest-derived tumors, pheochromocytomas and paragangliomas (Qin et al. 2010, Yao et al. 2010, Neumann et al. 2011), and have also been detected in renal cell carcinomas (Qin et al. 2014). Genes involved in susceptibility to pheochromocytomas and renal cancers are also mutated in other malignancies. To determine whether TMEM127 mutations also predispose to cancers affecting the pediatric population, herein, we investigated the integrity of TMEM127 in 155 samples of various cancer types from patients younger than 18 years of age. One group comprised 16 gastrointestinal stromal tumor samples, four germline and 12 tumors, from 13 patients. A second group encompassed germline DNA from 139 pediatric patients and included 53 hematological malignancies (39 acute lymphoid leukemias, three acute myeloid leukemias, five Hodgkin's and six non-Hodgkin's lymphomas), 22 osteosarcomas, 16 CNS tumors (five medulloblastomas, one astrocytoma, two gliomas, one craniopharyngioma, one atypical teratoid rhabdoid tumor, and five with unspecified histology), 12 germ cell tumors, eight Ewing's sarcomas, six neuroblastic tumors, five Wilms' tumors, four retinoblastomas, three rhabdomyosarcomas, three liver tumors (two hepatoblastomas and one hepatocarcinoma), one synovial sarcoma, one fibrosarcoma, one mesothelioma, one adrenocortical carcinoma, one desmoid tumor, one non-Langerhans histiocytosis, and one primitive myxoid mesenchymal tumor of nasal arch. Three patients had more than one tumor. Informed consent was obtained from all patients (approved by UTHSCSA and NIH IRB committees) and sequencing of the TMEM127 coding region was performed as described previously (Yao et al. 2010). Two germline TMEM127 missense variants were detected: c. $67 \mathrm{C}>\mathrm{A}$, p.Leu23Met, a novel variant, in one patient with Ewing's sarcoma and c.268G $>$ A, p.Val90Met in one case of craniopharyngioma (Fig. 1). The Val90Met variant has been previously reported in pheochromocytomas (Qin et al. 2010, Abermil et al. 2012), and has also been listed in the NHLBI Exome Sequencing Project and the Exome Aggregation Consortium, Cambridge, MA, USA (URL: http://exac.broadinstitute.org; March, 2015), two reference databases that include both healthy and disease cohorts, at 0.28 and $0.08 \%$ minor allele frequency (rs121908823) respectively. The patient with Ewing's sarcoma carried an EWSR1 translocation that has been previously implicated in this tumor's pathogenesis (Tsokos et al. 2012). Tumor tissue was not available from either patient for loss of heterozygosity analysis. No family history of cancers, pheochromocytoma, or paraganglioma was reported in these two cases and DNA from parents was not available for testing. No pathogenic variants were detected in the remaining samples. Previously, we found that ectopic expression of several mutant TMEM127 constructs can lead to a diffuse subcellular distribution of the protein, in contrast with the punctate, endomembrane-associated appearance of the WT TMEM127 product (Yao et al. 2010, Qin et al. 2014). To determine whether the variants detected in this study had aberrant distribution, we engineered TMEM127 constructs expressing these changes fused to the GFP protein as reported previously (Qin et al. 2014). We found that subcellular localization of the constructs was similar to that of WT TMEM127 when transfected into HeLa cells (data not shown). These findings indicate either that Leu23Met and Val90Met disrupt a function of TMEM127 that is independent of its membrane association or that they are not pathogenic. Currently, there are no established downstream studies to test other functions of TMEM127.

We also interrogated publicly available databases of sequence data from cancers, including The Cancer Genome Atlas (TCGA, NIH, USA) and the Catalog of Somatic Mutations in Cancer (COSMIC, Sanger Institute, UK). In these predominantly adult cancer cohorts, we identified 46 somatic TMEM127 mutations, some of which were recurrent and/or predicted to be pathogenically relevant, across multiple tumor types (Fig. 1). Intriguingly, these variants were entirely non-overlapping with

Published by Bioscientifica Ltd 
Germline TMEMI27 mutations

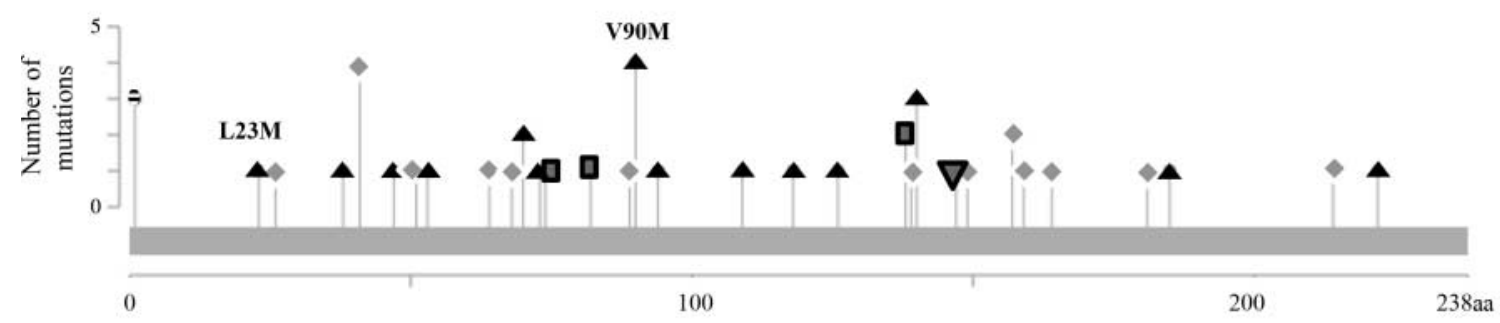

Somatic TMEM127 mutations

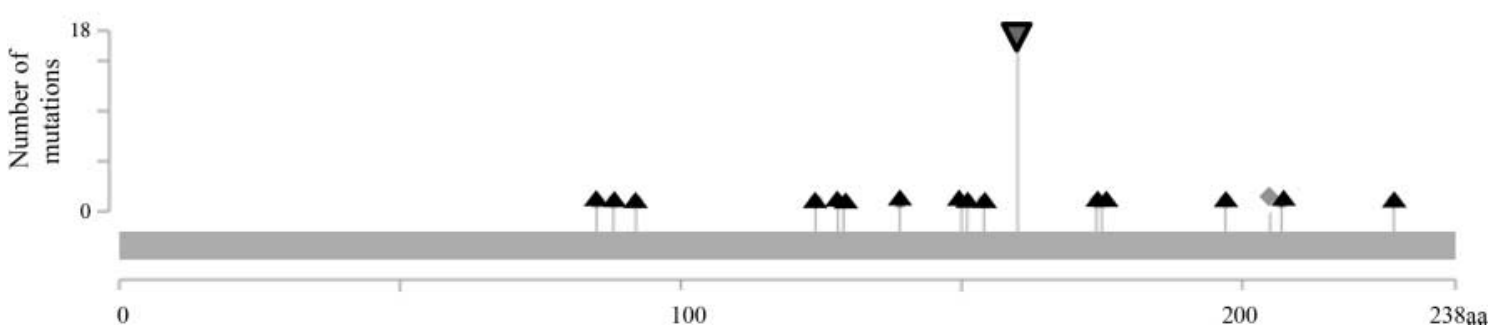

\section{Figure 1}

'Lollipop' plot displaying the number (vertical axis) and position of TMEM127 gene mutations along its protein sequence. The upper panel depicts germline mutations. The two mutations found in this study are indicated. The Val90Met (V90M) variant has been found previously in three pheochromocytomas. The lower panel shows somatic mutations found in breast, brain, liver, pancreas, skin, stomach, and endometrial cancers from

germline mutations reported in pheochromocytomas and renal cancer. The results of our study indicate that the overall contribution of TMEM127 to pediatric cancer predisposition is limited, if present at all, although the number of samples tested within individual tumor types was small. This finding may not be entirely surprising given that previously reported germline TMEM127 mutations occur predominantly in adult patients (Toledo et al. 2014). However, further studies will be necessary to establish whether somatic TMEM127 variants have functional significance in pediatric or adult cancers.

E King ${ }^{1}$ Y Qin ${ }^{1}$ R A Toledo ${ }^{1}$ A $\mathrm{Luo}^{1}$ $\mathrm{E} \mathrm{Ball}^{2}$ F R Faucz ${ }^{2}$ K A Janeway ${ }^{3}$ C A Stratakis ${ }^{2}$

G E Tomlinson ${ }^{4,5,6}$

P L M Dahia ${ }^{1,5,6}$ publicly available databases searchable at the Memorial Sloan Kettering Cancer Center cBioPortal for Cancer Genomics site (http://www.cbioportal. org/public-portal/). Key: missense mutations, triangles; nonsense or frameshift mutations, diamonds; splice mutations, squares; inframe insertions or deletions, inverted triangles; start codon loss/change, circles. The plot was designed using the Mutation Mapper tool of the cBioPortal.

${ }^{1}$ Department of Medicine, University of Texas Health Science Center at San Antonio (UTHSCSA),

San Antonio, Texas, 78229, USA

${ }^{2}$ Program on Developmental Endocrinology and Genetics, National Institutes of Health, Bethesda, Maryland, USA

${ }^{3}$ Department of Pediatric Oncology, Harvard Medical School, Dana-Farber Children's Hospital Cancer Center, Boston, Massachusetts, USA

${ }^{4}$ Department of Pediatrics, UTHSCSA, San Antonio,

Texas, USA

${ }^{5}$ Greehey Children Cancer Research Institute at UTHSCSA, San Antonio, Texas, USA

${ }^{6}$ Cancer Therapy and Research Center at UTHSCSA, San Antonio, Texas, USA

(Correspondence should be addressed to P L M Dahia; email: dahia@uthscsa.edu)

Declaration of interest

The authors declare that there is no conflict of interest that could be perceived as prejudicing the impartiality of the research reported.

Published by Bioscientifica Ltd. 


\section{Funding}

Funding support was received from the Voelcker Fund, Cancer Prevention and Research Institute of Texas (CPRIT), Department of Defense (DOD), Alex's Lemonade Stand Cancer Foundation, and the Greehey Childhood Cancer Research Institute (GCCRI) to P L M Dahia; Hyundai Hope on Wheels, St Baldrick's Foundation Infrastructure grant, and the GCCRI to G E Tomlinson; and the Intramural Research Program of the Eunice Kennedy Shriver National Institute of Child Health and Human Development (NICHD) to C A Stratakis. Images were generated in the Core Optical Imaging Facility, which is supported by UTHSCSA, NIH-NCI P30 CA54174 (CTRC at UTHSCSA), and NIH-NIA P01AG19316.

\section{Acknowledgements}

The authors are grateful to Nicole Paes Morales for technical assistance.

\section{References}

Abermil N, Guillaud-Bataille M, Burnichon N, Venisse A, Manivet P, Guignat L, Drui D, Chupin M, Josseaume C, Affres H et al. 2012 TMEM127 screening in a large cohort of patients with pheochromocytoma and/or paraganglioma. Journal of Clinical Endocrinology and Metabolism 97 E805-E809. (doi:10.1210/jc.2011-3360)
Neumann HP, Sullivan M, Winter A, Malinoc A, Hoffmann MM, Boedeker CC, Bertz H, Walz MK, Moeller LC, Schmid KW et al. 2011 Germline mutations of the TMEM127 gene in patients with paraganglioma of head and neck and extraadrenal abdominal sites. Journal of Clinical Endocrinology and Metabolism 96 E1279-E1282. (doi:10.1210/jc.2011-0114)

Qin Y, Deng Y, Ricketts CJ, Srikantan S, Wang E, Maher ER \& Dahia PL 2014 The tumor susceptibility gene TMEM127 is mutated in renal cell carcinomas and modulates endolysosomal function. Human Molecular Genetics 23 2428-2439. (doi:10.1093/hmg/ddt638)

Qin Y, Yao L, King EE, Buddavarapu K, Lenci RE, Chocron ES, Lechleiter JD, Sass M, Aronin N, Schiavi F et al. 2010 Germline mutations in TMEM127 confer susceptibility to pheochromocytoma. Nature Genetics $\mathbf{4 2}$ 229-233. (doi:10.1038/ng.533)

Toledo SP, Lourenco DM Jr, Sekiya T, Lucon AM, Baena RC, Castro CC, Bortolotto LA, Zerbini MC, Siqueira SA, Toledo RA et al. 2014 Penetrance and clinical features of pheochromocytoma in a six-generation family carrying a germline TMEM127 mutation. Journal of Clinical Endocrinology and Metabolism 100 E308-E318. (doi:10.1210/jc.2014-2473)

Tsokos M, Alaggio RD, Dehner LP \& Dickman PS 2012 Ewing sarcoma/ peripheral primitive neuroectodermal tumor and related tumors. Pediatric and Developmental Pathology 15 108-126. (doi:10.2350/11-081078-PB.1)

Yao L, Schiavi F, Cascon A, Qin Y, Inglada-Perez L, King EE, Toledo RA, Ercolino T, Rapizzi E, Ricketts CJ et al. 2010 Spectrum and prevalence of FP/TMEM127 gene mutations in pheochromocytomas and paragangliomas. Journal of the American Medical Association 304 2611-2619. (doi:10.1001/jama.2010.1830)

Received in final form 12 March 2015

Accepted 13 March 2015

Made available online as an Accepted Preprint

13 March 2015
(C) 2015 Society for Endocrinology Printed in Great Britain 